02

\title{
Угловые зависимости интенсивности комбинационного рассеяния света на поляритонах в кристалле фосфида галлия
}

\author{
(C) А.В. Иго \\ Ульяновский государственный университет, \\ 432063 Ульяновск, Россия \\ e-mail: igoalexander@mail.ru
}

Поступила в редакцию 01.11.2018 г.

В окончательной редакции 22.02.2019 г.

Принята к публикации 15.03.2019 г.

\begin{abstract}
Измерено комбинационное рассеяния света на фононах и поляритонах в образце фосфида галлия. Для возбуждения использовался несфокусированный пучок одномодового лазера $532 \mathrm{~nm}$. Сбор рассеянного излучения производился с помощью передвижного зеркала малого диаметра, что позволило измерять спектры рассеянного света в диапазоне углов рассеяния $0.6^{\circ}-8^{\circ}$ с угловой суммарной расходимостью $0.4^{\circ}$. Для разных кристаллографических направлений измерялись интенсивности поляризованных компонент комбинационного рассеянного света на продольных, поперечных фононах и поляритонах в области сильной дисперсии поляритонной ветви для трех фиксированных аксиальных углов рассеяния. Компоненты рассеяния на продольных оптических фононах и поляритонах имеют сильную зависимость от кристаллографического направления как и предсказывает теория, а компонента рассеяния на поперечных оптических фононах не зависела от кристаллографического направления. Обнаружено, что интенсивность рассеяния на поперечных оптических фононах коррелирует с шириной спектральной линии рассеяния на поляритоне. Предложен механизм, объясняющий эту корреляцию.
\end{abstract}

Ключевые слова: поляритон, фонон, комбинационное рассеяние света, ангармонизм

DOI: $10.21883 /$ OS.2019.08.48033.316-18

\section{Введение}

Комбинационное рассеяние света (КРС) широко применяется для исследования поляритонов в кристаллах [1]. Проведенные исследования показывают, что теория $[1,2]$ в основном правильно описывает возникновение и существование в полярных кристаллах гибридных возбуждений, образованных квантами колебаний кристаллической решетки при взаимодействии с электромагнитным полем. Эксперимент подтвердил вид дисперсионных зависимостей поляритонов в кристаллах [1], зависимость частоты поляритона от направления в одноосном кристалле [3]. При измерении КРС на поляритонах замечено, что ширина и форма спектральной линии зависят от частоты и волнового вектора поляритона. Результаты таких исследований для кристаллов GaP подробно отражены в работе [4], где рассмотрены вклады оптической и механической ангармоничностей в ширину спектральной линии.

Ширина спектральной линии КРС, обычно однозначно связанная с временем жизни элементарного возбуждения, в случае рассеяниях на поляритонах зависит от угла рассеяния [4]. А именно, измеренная ширина спектральной линии содержит в себе часть, связанную собственно с временем жизни поляритона с определенным волновым вектором, и часть, обусловленную тем, что при фиксировании угла рассеяния (и сканирования по частоте) волновой вектор поляритона не остается фиксированным и изменяется в некотором диапазоне.
В $[1,2]$ теоретически показано, что если в качестве искомой частоты брать центр спектральной линии (даже искаженной), то эти данные хорошо соответствуют теории дисперсии поляритонов.

Таким образом, форма и ширина спектральной линии зависят от техники и методики измерений [4], длины волны лазерного возбуждающего излучения [1,5], а их зависимость от угла рассеяния (или пересчитанные на частоту поляритона) всегда имеют особенности, вносимые методикой эксперимента. Исследования дисперсии и симметрии поляритонов в средах, важных с точки зрения практического применения, продолжаются [6].

Первой целью настоящей работы являлось измерение КРС поляритонов по методике, отличающейся от описанных в обзорах, оценка погрешности измерения по представленной методике и сопоставление полученных данных с известными зависимостями.

Вторая цель работы состояла в измерении зависимости интенсивности КРС от кристаллографического направления и поляризаций падающего и рассеянного света, что обычно называется поляризационными измерениями. Теоретически [7] интенсивность рассеянного света на фононах определяется тензором КРС и может быть рассчитана для любой конфигурации, используемой в эксперименте. Поворачивая кристаллический образец вокруг определенного выбранного направления, можно получить кривую интенсивности КРС в зависимости от угла поворота. В совершенных кристаллах теоретическая и экспериментальные кривые совпадают $[8,9]$, но в 
кристаллах с дефектами, с сильным рассеянием фононов наблюдаются характерные отличия [9]. Представляется интересным определить, как влияет на угловые зависимости интенсивности КРС на поляритонах их сильное затухание по сравнению с обычными фононами.

\section{Эксперимент}

Схема эксперимента представлена на рис. 1. Пластина GaP 1 толщиной $0.36 \mathrm{~mm}$, ориентированная плоскостью (111) перпендикулярно направлению $z$, закреплена на держателе 2 с отверстием в центре. Держатель свободно поворачивается вокруг оси $z$ на $360^{\circ}$ и имеет шкалу с шагом $1^{\circ}$ для определения угла $\varphi$. Образец освещался несфокусированным пучком одномодового лазера CPDS532M 3, диаметр лазерного луча $0.6 \mathrm{~mm}$, угловая расходимость менее $0.0012 \mathrm{rad}$, длина волны $532 \mathrm{~nm}$, мощность $25 \mathrm{~mW}$. Рассеянный свет собирался повернутым круглым зеркалом 4 диаметром $2 \mathrm{~nm}$, расположенным на подвижном держателе, который позволяет перемещать зеркало по оси $y$. Смещение зеркала относительно центра лазерного луча измерялось по микрометрической шкале с погрешностью $0.05 \mathrm{~nm}$. Расстояние от зеркала до образца $L_{0}=57 \mathrm{~nm}$. Отраженный от зеркала свет проходил через регулируемую диафрагму 5, поляризатор 6 и собирался линзой 7 на входную щель модернизированного спектрометра ДФС-52 с ФЭУ Н6240-01 8. Лазерный луч поляризован в направлении $x$ (перпендикулярно рисунку) или $y$, поворот осуществлялся полуволновой пластинкой 9.

Измерения спектров КРС проводились в диапазоне $290-425 \mathrm{~cm}^{-1}$ с шагом $0.5 \mathrm{~cm}^{-1}$ и спектральной шириной щели $1 \mathrm{~cm}^{-1}$. Угол рассеяния задавался держателем зеркала 4 и рассчитывался по координате смещения зеркала относительно лазерного луча $y_{i}$ как $\theta_{i}=y_{i} /\left(L_{0} n_{0}\right)$, где $n_{0}$ - показатель преломления GaP. Азимутальный

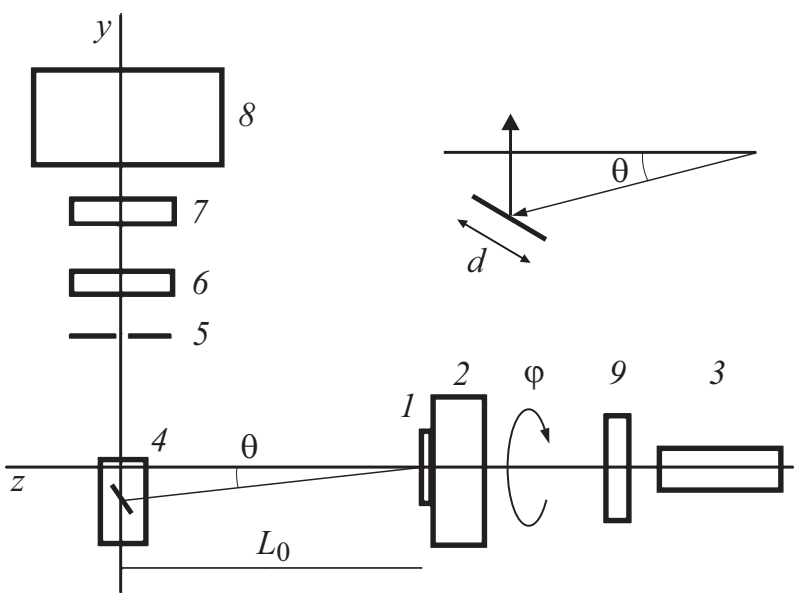

Pис. 1. Схема эксперимента для измерения угловой зависимости КРС на поляритонах: 1 - образец, 2 - держатель, 3 - лазер, 4 - зеркало размером $d, 5$ - диафрагма, $6-$ поляризатор, 7 - линза, 8 - спектрометр, 9 - пластинка $\lambda / 2$.

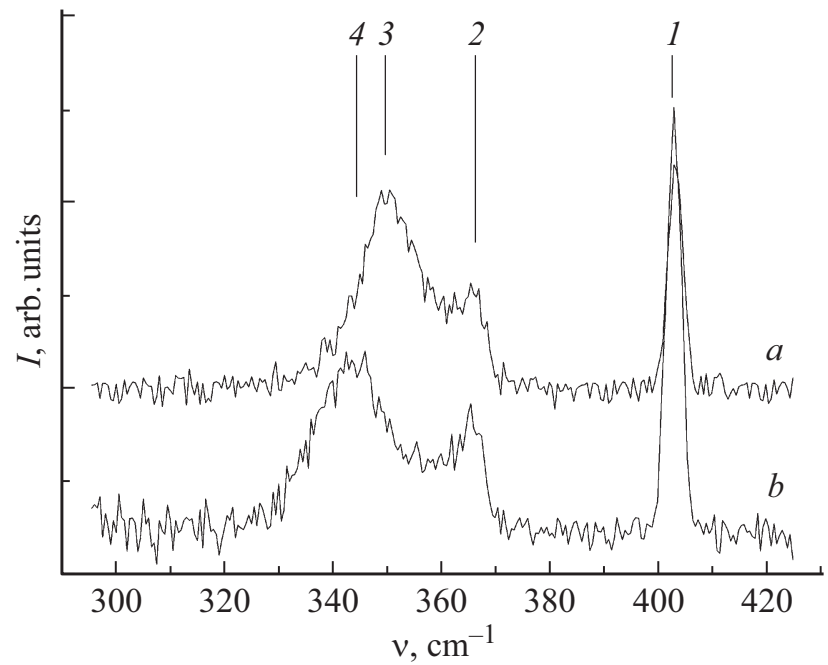

Pис. 2. Спектры КРС для двух углов рассеяния: $\theta=1.2^{\circ}(a)$, $0.7^{\circ}(b)$. Пояснения в тексте.

угол $\varphi$ отсчитывался от направления оси $x$. На рис. 2 представлены спектры КРС образца при $\varphi=270^{\circ}$, $\theta=1.2^{\circ}(a)$ и $0.7^{\circ}(b), x$-поляризации падающего луча, рассеянный луч без поляризатора. Пики на спектрах при $403 \mathrm{~cm}^{-1}$ соответствуют рассеянию света на LOфононах - 1, при $465 \mathrm{~cm}^{-1}$ на ТО-фононах - 2, при $350 \mathrm{~cm}^{-1}$ и $344 \mathrm{~cm}^{-1}$ на поляритонах - 3 и 4 соответственно. Положение пика поляритона зависит от угла рассеяния.

Измерены спектры для углов рассеяния в диапазоне $0.6^{\circ}-8^{\circ}$ при $\varphi=270^{\circ}$, определены положение максимума и ширина спектральной линии поляритона. График зависимости ширины спектральной линии КРС на поляритонах от частоты поляритона представлен на рис. $3, b$ в виде точек. Измеренные положения максимума спектральной линии поляритона в зависимости от угла рассеяния представлены на рис. 3, $a$ в виде дисперсионной зависимости. Волновой вектор поляритона $q$, соответствующий измеренному углу рассеяния $\theta$, определялся из закона сохранения импульса как описано в работе [1]:

$$
q^{2}=k_{i}^{2}+k_{s}^{2}-2 k_{i} k_{s} \cos \theta,
$$

где $k_{i}$ и $k_{s}$ - волновые векторы падающего и рассеянного света. В образце величина $k_{i}$ задается длиной волны лазера $\lambda_{0}=532 \mathrm{~nm}$ (волновым числом $v_{0}=1 / \lambda_{0}$, измеряемым спектрометром) и показателем преломления света $\mathrm{GaP}$ на длине волны лазера $n_{0}=3.492$ [10]:

$$
k_{i}=2 \pi v_{0} n_{0}
$$

Волновой вектор рассеянного света $k_{s}$ зависит от значения показателя преломления света образца на частоте рассеянного света:

$$
k_{s}=2 \pi \nu n
$$



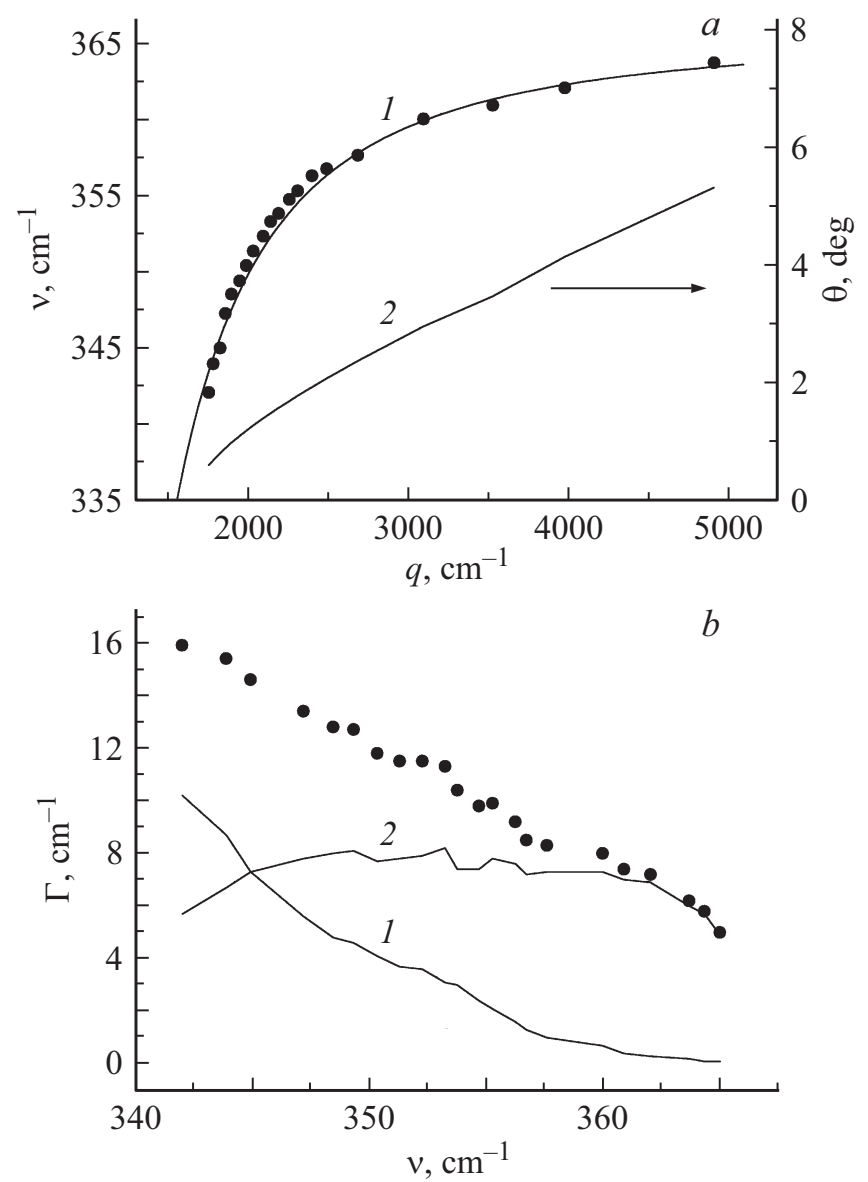

Рис. 3. Дисперсия поляритонов (a), ширина спектральной линии КРС на поляритоне $(b)$. Пояснения в тексте.

В линейном приближении

$$
n=n_{0}+\frac{\partial n}{\partial v} v
$$

По данным [10] для $\mathrm{GaP}$ в окрестности $532 \mathrm{~nm}$ $\frac{\partial n}{\partial v}=0.737 \cdot 10^{-4}$. Частота рассеянного света $v$ связана с частотой поляритона и частотой падающего света законом сохранения энергии:

$$
v=v_{0}-v(q),
$$

где $v(q)$ частота поляритона с волновым вектором $q$. Теоретическая дисперсионная зависимость поляритонов [11] следующая:

$$
\begin{aligned}
v^{2}(q)= & \frac{1}{2}\left(v_{\mathrm{LO}}^{2}+\frac{q^{2}}{\varepsilon_{\infty}}\right) \\
& -\frac{1}{2}\left(\left(v_{\mathrm{LO}}^{2}+\frac{q^{2}}{\varepsilon_{\infty}}\right)^{2}-4 \frac{q^{2}}{\varepsilon_{\infty}} v_{\mathrm{TO}}^{2}\right)^{1 / 2} .
\end{aligned}
$$

Совместное решение уравнений (1) и (2) позволило определить значения $q$ для набора углов рассеяния $\theta$, заданных в эксперименте. Результаты расчета приведены на рис. 3, $a$, кривая 2 . В расчетах использовались значения частот из эксперимента $v_{\mathrm{LO}}=403 \mathrm{~cm}^{-1}$, $v_{\text {ТО }}=465 \mathrm{~cm}^{-1}$ и $\varepsilon_{\infty}=9.09$ [11].

Были измерены интенсивности спектральных линий КРС в зависимости от азимутального угла $\varphi$ для значений угла рассеяния $\theta=0.9^{\circ}, 1.4^{\circ}, 2.0^{\circ}$. На рис. 4 точками представлены интенсивности линий рассеяния на LO-фононах $(a)$, поляритонах $(b)$ и TO-фононах $(c)$ для угла рассеяния $\theta=0.9^{\circ}$, угол $\varphi$ менялся в интервале $0^{\circ}-360^{\circ}$ с шагом $10^{\circ}$. На графиках интенсивности представлены в нормированном виде, в эксперименте интенсивность линии LO-фонона (в максимуме) в 1.9 раза больше интенсивности линии поляритона (в максимуме). Интенсивность рассеяния на ТО-фононе указана в долях от максимального значения интенсивности линии поляритона. Интенсивность линии считалась как площадь под спектральной кривой, расчет проводился с помощью программы Origin 8.

\section{Угловая зависимость интенсивности KPC}

В полярных кубических кристаллах интенсивность рассеянного света на LO- и TO-фононах в выбранном направлении может быть рассчитана теоретически [7], если известен тензор КРС кристалла $\hat{\mathbf{R}}$ и поляризация падающего $\mathbf{e}_{i}$ и рассеянного $\mathbf{e}_{s}$ света:

$$
I=A\left[\Sigma_{\rho, \sigma, \tau} e_{i}^{\rho} R_{\rho, \sigma}^{\tau} \xi^{\tau} e_{s}^{\sigma}\right]^{2} .
$$

Единичный вектор поляризации фонона $\boldsymbol{\xi}$ определяется углом рассеяния $\theta$, значениями $k_{i}$ и $k_{s}$ и $q$ из (1) и

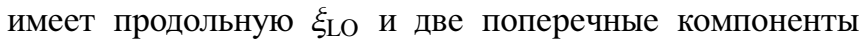
$\xi_{\mathrm{TO} 1}, \xi_{\mathrm{TO} 2}$. В лабораторной системе координат (рис.1) компоненты имеют вид

$$
\begin{gathered}
\xi_{\mathrm{LO}}=\left(0,-\frac{k_{s} \sin \theta}{q}, \frac{k_{i}-k_{s} \cos \theta}{q}\right), \quad \xi_{\mathrm{TO} 1}=(1,0,0), \\
\xi_{\mathrm{TO} 2}=\left(0,-\frac{k_{i}-k_{s} \cos \theta}{q},-\frac{k_{s} \sin \theta}{q}\right) .
\end{gathered}
$$

Вид тензора $\hat{\mathbf{R}}$ для $\mathrm{GaP}$ с симметрией кристаллической решетки $\mathbf{T}_{d}$ имеет одну независимую компоненту $d$ и в главных осях имеет вид [7]

$$
\hat{\mathbf{R}}=\left(\left(\begin{array}{lll}
0 & 0 & 0 \\
0 & 0 & d \\
0 & d & 0
\end{array}\right), \quad\left(\begin{array}{lll}
0 & 0 & d \\
0 & 0 & 0 \\
d & 0 & 0
\end{array}\right), \quad\left(\begin{array}{lll}
0 & d & 0 \\
d & 0 & 0 \\
0 & 0 & 0
\end{array}\right)\right)
$$

Преобразование $\hat{\mathbf{R}}$ в лабораторную систему координат, в которой направление [111] кристалла соответствует оси $z$, а направление [1ㅣㅣ - оси $y$, проводится матрицей поворота $U_{111}$, поворот вокруг оси $z$ на произвольный угол $\varphi$ выполняется матрицей $U_{\varphi}$, а суммарное 
последовательное вращение - матрицей $U$ :

$$
\begin{gathered}
U=U_{\varphi} U_{111}, \quad U_{111}=\left(\begin{array}{ccc}
\frac{-1}{\sqrt{6}} & \frac{-1}{\sqrt{6}} & \frac{2}{\sqrt{6}} \\
\frac{1}{\sqrt{2}} & \frac{-1}{\sqrt{2}} & 0 \\
\frac{1}{\sqrt{3}} & \frac{1}{\sqrt{3}} & \frac{1}{\sqrt{3}}
\end{array}\right), \\
U_{\varphi}=\left(\begin{array}{ccc}
\cos \varphi & -\sin \varphi & 0 \\
\sin \varphi & \cos \varphi & 0 \\
0 & 0 & 1
\end{array}\right) .
\end{gathered}
$$

Из положения повернутого на произвольный угол кристалла преобразуем компоненты вектора поляризации фонона в главные оси кристалла и найдем значения тензора КРС для рассеяния на продольных и поперечных фононах в главных осях:

$$
\begin{gathered}
R_{\mathrm{LO}}=\hat{R} U^{\mathrm{T}} \xi_{\mathrm{LO}}, \quad R_{\mathrm{TO} 1}=\hat{R} U^{\mathrm{T}} \xi_{\mathrm{TO} 1}, \\
R_{\mathrm{TO} 2}=\hat{R} U^{\mathrm{T}} \xi_{\mathrm{TO} 2},
\end{gathered}
$$

где $U^{\mathrm{T}}$ - транспонированная матрица $U$. Задавая поляризацию падающего и рассеянного света $\mathbf{e}_{i}, \mathbf{e}_{s}$ из (3), получим для интенсивности рассеяния вперед под углом $\theta$ на LO-и TO- фононах для кристалла, повернутого на произвольный угол $\varphi$ :

$$
\begin{gathered}
I^{\mathrm{LO}}(\varphi)=\left[\mathbf{e}_{i} U R_{\mathrm{LO}} U^{\mathrm{T}} \mathbf{e}_{s}\right]^{2}, \\
I^{\mathrm{TO}}(\varphi)=\left[\mathbf{e}_{i} U R_{\mathrm{TO} 1} U^{\mathrm{T}} \mathbf{e}_{s}\right]^{2}+\left[\mathbf{e}_{i} U R_{\mathrm{TO} 2} U^{\mathrm{T}} \mathbf{e}_{s}\right]^{2} .
\end{gathered}
$$

Расчет интенсивностей $I_{x x}^{\mathrm{LO}}(\varphi), I_{x x}^{\mathrm{TO}}(\varphi)$ по формуле (4) для заданных в эксперименте условий и поляризаций $e_{i}=e_{s}=e_{x}=(1,0,0), \theta=0.9^{\circ}$ представлен на рис. 4 сплошными линиями. Расчет проводился в программе Wolfram Mathematica.

\section{Обсуждение результатов эксперимента}

Эксперимент показывает сильную зависимость ширины спектральной линии поляритона от угла рассеяния (рис. $3, b$ ). Как было показано в [4], необходимо различать ширину спектральной линии поляритона, обусловленную конечным временем жизни, и дополнительную ширину, обусловленную особенностью измерения КРС в области сильной дисперсии. А именно, в области сильной дисперсии при измерении КРС дополнительная ширина спектральной линии определяется собственно дисперсией и угловым размером измерительного инструмента:

$$
\Delta v=\frac{\partial v}{\partial \theta} \Delta \theta,
$$

угол сбора рассеянного излучения в эксперименте (рис. 1):

$$
\Delta \theta_{1}=\frac{d}{L_{0}} \cos \left(\frac{\pi / 2-\theta}{2}\right) \cong 0.025 .
$$

С учетом угловой расходимости лазера, внутри кристалла $\Delta \theta=0.0074 \mathrm{rad}$. На рис. $3, b$ кривой 1 представлена зависимость (5), значения производной определялись по экспериментальным точкам (рис. 3,a). Сравнивая экспериментальную зависимость (точки) и кривую 1 , можно сделать вывод, что основной вклад в увеличение ширины спектральной линии поляритона происходит за счет $\Delta v$.

Разность между экспериментальными данными и кривой 1 представлена кривой 2 . Кривая имеет колоколообразную форму с максимумом в области $355 \mathrm{~cm}^{-1}$, где ранее было обнаружен локальный максимум, который в [4] был приписан механическому ангармонизму. Дисперсия поляритонов в области $355 \mathrm{~cm}^{-1}$ действительно отличается от гармонического приближения. На рис. $3, a$ видно, что в области $355 \mathrm{~cm}^{-1}$ экспериментальные точки не совпадают с кривой 1 , построенной по формуле (2).

Угловая зависимость интенсивности КРС, полученная в эксперименте, хорошо совпадает с теорией. В минимумах интенсивности (углы рассеяния $30^{\circ}, 150^{\circ}, 270^{\circ}$ на LO-фононах (рис. $4, a$ ) и $90^{\circ}, 210^{\circ}, 330^{\circ}$ для поляритонов (рис. $4, b)$ ) нулевые значения интенсивности в эксперименте не наблюдаются. Это связано с неидеальностью поляризатора, эллиптичностью поляризации лазера, точностью выставления угла поворота - все вместе определяет погрешность измерений. Минимальное экспериментальное значение интенсивности составляет 0.15 от ее максимального значения для LO-рассеяния и 0.18 для рассеяния на поляритонах.

Рассеяние на ТО-фононах (пик 2 на рис. 2) в эксперименте не зависит от угла поворота (рис. 4,c) и имеет постоянную интенсивность $0.17 \pm 0.02$ от интенсивности поляритона в максимуме. В эксперименте по КРС на поляритонах такое ТО-рассеяние присутствует всегда $[1,5]$. Обычно оно объясняется рассеянием назад от задней стенки образца (на угол $\theta=\pi$, а рассеяние на любой угол $\theta \gtrsim 8^{\circ}$ будет с частотой $\left.v=v_{\text {то }}\right)$. Такое объяснение, возможно - действительно, формула (4) дает изотропное рассеяние назад: $I_{x x}^{\mathrm{LO}}(\varphi)=0.5, I_{x x}^{\mathrm{TO}}(\varphi)=0.5$, и с учетом потери при отражении $(R \simeq 0.31[10])$ при минимальном поглощении света в образце. В данном случае при $532 \mathrm{~nm}$ поглощение света в образце значительно $\alpha=360 \mathrm{~cm}^{-1}[10]$. Рассеяние назад на LO-фононах отсутствует, так как изотропная составляющая (минимальное значение) его равна 0.15 и, таким образом, она меньше, чем изотропная составляющая рассеяния на поляритонах $(0.18)$, которая точно является погрешностью эксперимента. Таким образом, это ТО-рассеяние не может быть рассеянием назад, так как нет рассеяния назад на LO.

Другим объяснением наличия изотропного ТО-рассеяния в эксперименте является предложенный в [9] механизм. Наличие ангармонического взаимодействия приводит к тому, что фонон или в данном случае поляритон уже не является плоской волной, а в соответствии с подходом квантовой механики представляет собой сумму плоской первичной волны и сферической 

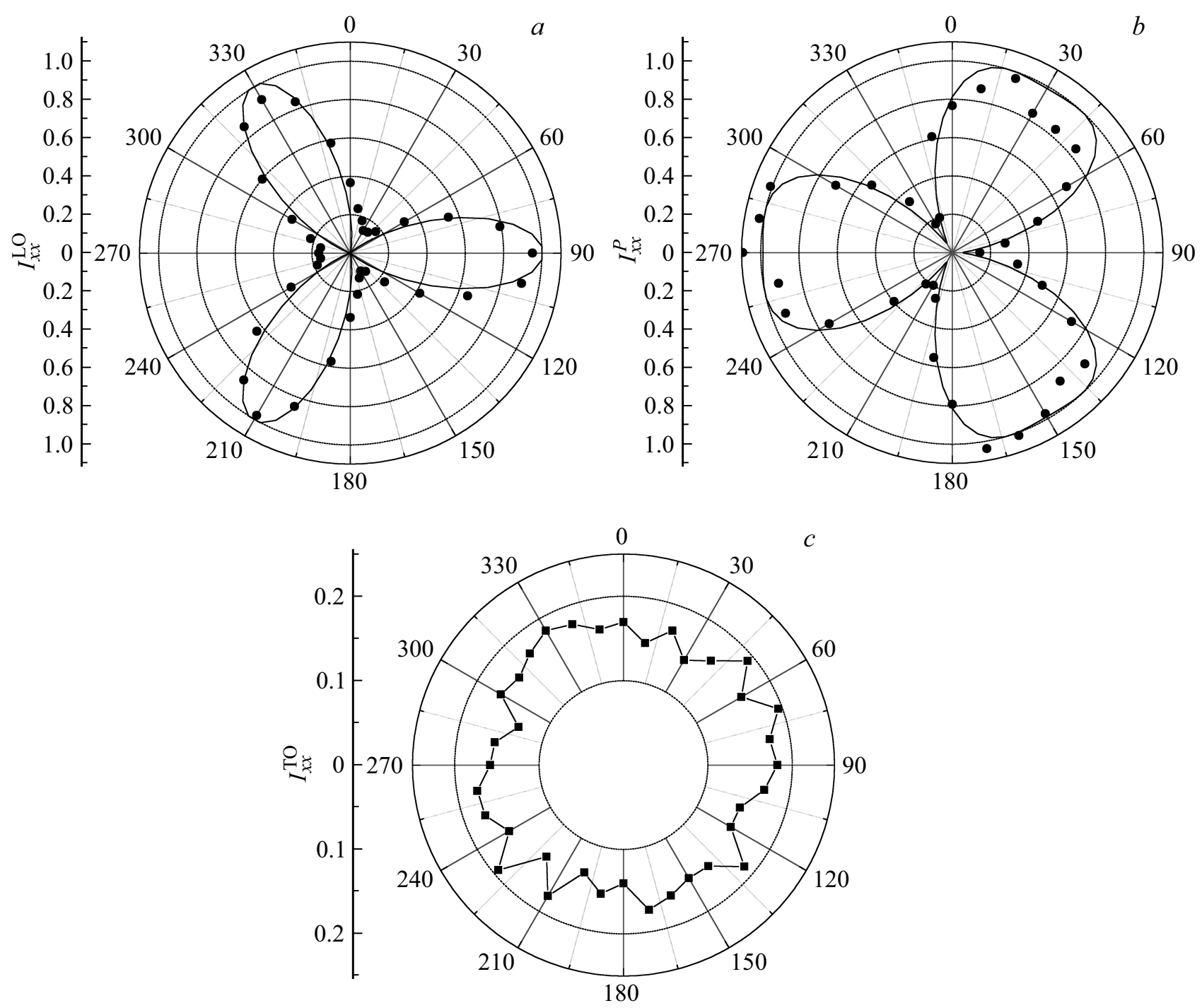

Рис. 4. Угловая зависимость интенсивности КРС в кристалле $\mathrm{GaP}$, угол поворота кристалла $\varphi$ обозначен на рис. $1:(a) I_{x x}^{\mathrm{LO}}(\varphi)$, точки - эксперимент, сплошная линия - расчет по формуле $(4),(b) I_{x x}^{P}(\varphi)$, точки - эксперимент, сплошная линия — расчет по формуле $(4),(c) I_{x x}^{\mathrm{TO}}(\varphi)$, экспериментальные точки соединены линией для наглядности.

рассеянной:

$$
\Psi=e^{i q z}+\frac{f}{r} e^{i q r}
$$

Первое слагаемое описывает невзаимодействующий поляритон, распространяющийся вдоль заданного направления $z$, и свет рассеивается на нем на частоте поляритона $v(q)$. Амплитуда рассеяния $f$ во втором слагаемом определяет интенсивность рассеянного света от сферически расходящегося колебания, а частота рассеянного света соответственно $\nu_{\text {Tо }}$. Для сильного ангармонического взаимодействия амплитуда рассеяния должна возрастать и соответственно должна увеличиться интенсивность ТО-рассеяния. Измерение отношений интенсивностей ТО- и поляритонного рассеяния для углов рассеяния ( частоты поляритона) $0.9^{\circ}\left(346 \mathrm{~cm}^{-1}\right), 1.4^{\circ}\left(352 \mathrm{~cm}^{-1}\right), 2.0^{\circ}\left(356 \mathrm{~cm}^{-1}\right)$ дает $0.17 \pm 0.02,0.18 \pm 0.02,0.19 \pm 0.02$. Такое увеличение амплитуды рассеяния коррелирует с увеличением ширины спектральной линии КРС на поляритонах с 7 до $8 \mathrm{~cm}^{-1}$ (кривая 2 на рис. $3, b$ ).

Рассеяние на LO-колебаниях имеет очень узкую спектральную линию КРС. Измеренная в эксперименте величина составляла $3.4 \mathrm{~cm}^{-1}$, но это завышенное значение, так как спектральная ширина щели составляла $1 \mathrm{~cm}^{-1}$ и значение $3.4 \mathrm{~cm}^{-1}$ это свертка собственной ширины спектральной линии с гауссовой аппаратной функцией щели спектрометра. Более того, и вид спектральной линии соответствует гауссову профилю. Узкая линия в спектре естественно означает, что для LO ангармоническое взаимодействие минимально и фонон хорошо описывается невзаимодействующей плоской волной. Форма спектральной линии рассеяния на поляритонах имеет характерный лоренцев профиль, означающий, что она не искажена аппаратной функцией щели спектрометра. 


\section{Выводы}

Применена новая методика измерения КРС на фононах и поляритонах в образце GaP. Для возбуждения КРС использовался несфокусированный пучок одномодового лазера $532 \mathrm{~nm}$, а для сбора излучения использовано передвижное зеркало малого диаметра. Полученные спектры и зависимости спектральных параметров КРС от угла рассеяния в основном совпадают с ранее полученными значениями.

Проведены измерения зависимости интенсивности КРС от кристаллографического направления. Исследовались угловые зависимости (в азимутальной плоскости) поляризованных компонент интенсивности рассеянного света на LO- и TO-фононах и поляритонах (в области сильной дисперсии поляритонной ветви для трех фиксированных аксиальных углов рассеяния). Экспериментальные данные сопоставлены с теоретическими зависимостями. Наблюдаемые в эксперименте угловые зависимости поляритонов правильно описываются тензором комбинационного рассеяния света.

Обнаружено, что в области сильной дисперсии поляритонной ветви величина ангармонического взаимодействия поляритона коррелирует с интенсивностью изотропного рассеяния. Полученный результат является новым. Мерой ангармонизма является ширина спектральной линии КРС на поляритоне, а величину изотропного рассеяния в эксперименте определяли по угловым зависимостям интенсивности КРС.

Полученные результаты могут быть использованы для измерения величин ангармонизма, дефектности или других несовершенств в кристаллах.

\section{Конфликт интересов.}

Автор заявляет, что у него нет конфликта интересов.

\section{Список литературы}

[1] Поливанов Ю.Н. // УФН. 1978. Т. 126. № 2. С. 185

[2] Benson H.J., Mills D.L. // Phys. Rev. B. 1970. V. 1. N 12. P. 4835.

[3] Irmer G., Roder C., Himcinschi C., Kortus J. // Phys. Rev. B. 2013. V. 88. N 10. P. 104303.

[4] Маврин Б.Н., Стерин Х.Е. // Современные проблемы спектроскопии комбинационного рассеяния света. М.: Наука, 1978. С. 303.

[5] Nicola J.H., Liete R.C.C. // Phys. Rev. B. 1975. V. 11. N 2. P. 798.

[6] Kojima S., Kanehara K., Hoshina T., Tsurumi T. // Jpn. J. Appl. Phys. 2016. V. 55. N 10. P. 2.

[7] Loudon R. // Adv. Phys. 1964. V. 13. P. 423.

[8] Munisso M.C., Zhy W., Pezzotti G. // Phys. Stat. Sol. B. 2009. V. 246. N 8. P. 1893.

[9] Иго А.В. // Опт. и спектр. 2018. Т. 125. В. 1. С. 25.

[10] Гавриленко В.И., Грехов А.М., Корбутяк Д.В., Литовченко В.Г. Оптические свойства полупроводников. Киев: Наукова Думка, 1987. С. 607.

[11] Ushioda S., McMullen J.D. // Sol. St. Comm. 1972. V. 11. P. 299. 